\title{
The genetic basis of hybrid male sterility in sympatric Primulina species
}

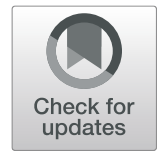

Chen Feng ${ }^{1,2}$, Huiqin $\mathrm{Yi}^{1,3}$, Lihua Yang ${ }^{1,2}$ and Ming Kang ${ }^{1,2^{*}}$ (D)

\begin{abstract}
Background: Sympatric sister species provide an opportunity to investigate the genetic mechanisms and evolutionary forces that maintain species boundaries. The persistence of morphologically and genetically distinct populations in sympatry can only occur if some degree of reproductive isolation exists. A pair of sympatric sister species of Primulina (P. depressa and P. danxiaensis) was used to explore the genetic architecture of hybrid male sterility.

Results: We mapped one major- and seven minor-effect quantitative trait loci (QTLs) that underlie pollen fertility rate (PFR). These loci jointly explained $55.4 \%$ of the phenotypic variation in the $F_{2}$ population. A BatesonDobzhansky-Muller (BDM) model involving three loci was observed in this system. We found genotypic correlations between hybrid male sterility and flower morphology, consistent with the weak but significant phenotypic correlations between PFR and floral traits.

Conclusions: Hybrid male sterility in Primulina is controlled by a polygenic genetic basis with a complex pattern. The genetic incompatibility involves a three-locus BDM model. Hybrid male sterility is genetically correlated with floral morphology and divergence hitchhiking may occur between them.
\end{abstract}

Keywords: Genetic architecture, Reproductive isolation, Hybrid male sterility, QTL, Primulina

\section{Background}

Barriers to genetic exchange between diverging populations promote reproductive isolation and, ultimately, speciation. Genetic divergence of barriers between populations involves the evolution of prezygotic and postzygotic isolation [1-3]. Individual isolation barriers may be incomplete, while sequentially acting barriers restrict gene flow between diverging lineages. Reproductive isolation components that act early are often thought to contribute more to total isolation than late-acting postzygotic barriers $[4,5]$. However, postzygotic isolation is undoubtedly a major factor in population divergence in

\footnotetext{
*Correspondence: mingkang@scbg.ac.cn

${ }^{1}$ Key Laboratory of Plant Resources Conservation and Sustainable Utilization,

South China Botanical Garden, Chinese Academy of Sciences, Guangzhou 510650, China

${ }^{2}$ Center of Conservation Biology, Core Botanical Gardens, Chinese Academy of Sciences, Guangzhou 510650, China

Full list of author information is available at the end of the article
}

the majority of systems [6]. Hybrid sterility and inviability are common forms of intrinsic barriers of postzygotic isolation, which lead to genetic differentiation and maintain species integrity [7-9].

It has been hypothesized that postzygotic reproductive isolation accumulates via increasing genomic incompatibilities [10-12], and that it is the by-product of genomic divergence [13]. Various genetic mechanisms of hybrid male sterility were proposed [14]. Of them, the Bateson-Dobzhansky-Muller (BDM) model predicts that hybrid sterility is caused by the accumulative negative interaction of two or more loci. For example, a single pair of heterospecific loci results in nearly complete hybrid male sterility in Mimulus [15]. Cytoplasmic male sterility (CMS) is another common genic incompatibility mechanism, which results from a mitochondrial-nuclear mismatch. The hybrid CMS mechanism has been widely studied in model species such as 
rice [16] and Arabidopsis [17]. In rice, a few genes causing hybrid sterility have been cloned [17-19] and several hypotheses have been proposed to explain the genetic mechanisms of hybrid sterility, such as the "duplicate gametophytic lethal model" [20] and the "onelocus allelic interaction model" [21]. In contrast to these simple models, many other studies demonstrated that hybrid sterility is a complex phenotype and is controlled by complicated mechanisms involving multiple loci. For example, empirical works in Drosophila and mice have revealed that hybrid sterility is highly polygenic and complex [22-24]. It is likely that genetic bases of hybrid sterility are extremely diverged in different systems. With genomic data increasingly accessible for non-model organisms [25], thousands of loci are easily identified for many lineages, potentially providing unprecedented power to study genetic mechanisms of hybrid sterility.

Theoretically, if there is gene flow between diverging species, tightly linked loci have larger selection coefficients than single loci $[26,27]$. This was evidenced by many empirical studies, especially on Mimulus [28, 29]. Furthermore, genetic linkage will be increased between loci involved in adaptation and reproductive isolation [30-32]. Thus, a pleiotropic locus or tightly physically linked loci for adaptive traits and reproductive isolation would be the most probable way for reproductive isolation to arise. In plants, hybrid male sterility often occurs concomitantly with pleiotropic effects on floral morphology (reviewed in [33]). In Mimulus, hybrid male fertility is significantly correlated to corolla size phenotypically $[34,35]$. In addition to pleiotropy, local adapted adjacent populations may also become reproductively isolated through hitchhiking of ecological adaptation genes and hybrid incompatibility loci [36]. Quantitative trait locus (QTL) mapping is an efficient approach for detecting pleiotropic or tightly linked loci underlying phenotypically correlated traits, and it is one of the most important fundamental methods for further detecting the hitchhiking effect.

In this study, we focus on Primulina depressa and Primulina danxiaensis, a pair of sympatric sister species $(2 n=2 x=36) . P$. depressa is characterized as large and blue-purple flowers [37] while $P$. danxiaensis flowers are small and light-yellow [38], and before recent phylogenetic analysis, they were placed in two different genera [39]. Recent molecular dating revealed these two species diverged approximately 2.1 million years ago [40]. Our previous study [41] of this species pair found that flower morphological traits, as prepollination barriers, are likely subject to divergent selection. However, several lines of evidence suggest that postpollination isolation barriers are likely to play an important role in blocking hybridization between them, because of their sympatric distribution with overlapping flowering time and shared pollinators such as Amegilla spp., Bombus spp., and Nomia spp.. In our crossing experiments, hybrids of these sympatric sister species showed decreased male fertility. This indicats the potential postzygotic isolation barriers between $P$. depress $a$ and $P$. danxiaensis, and makes them an ideal system for investigation of the hybrid sterility mechanisms and their evolution.

Here, we estimated hybrid male sterility in the species divergence between these sympatric sister species through heterosis analysis. We conducted a QTL mapping analysis for hybrid pollen fertility with an $F_{2}$ population. We characterized the number, mode of action, digenic interactions, and phenotypic effects of loci that cause male sterility in $F_{2}$ hybrids. We then detected whether hybrid male sterility QTLs were coincident with other flower and leaf trait QTLs. Our main objectives are to dissect the genetic basis of hybrid fertility, which may play an important role in reproductive isolation in this system.

\section{Results}

Analysis of variance and correlations

The proportion of fertile and sterile pollen in all individuals was evaluated by testing pollen viability with 2,3,5triphenyltetrazolium chloride (TTC). Under normal cultivation conditions (excluding harsh environments such as drought, flood, and extremely high or low temperatures), the pollen of parental lines ( $P$. depressa and $P$. danxiaensis) was completely fertile (the pollen fertility rate [PFR] was about $95 \%$ ), and the pollen of $F_{1}$ hybrids was semi-fertile (53.02\%). The PFR of $\mathrm{F}_{2}$ individuals ranged from $0 \%$ (completely sterile) to $69.69 \%$, with an average of $41.17 \%$ (Fig. 1). In the $F_{2}$ population, the PFR exhibited continuous variation, with a novel class of individuals $(15.4 \%)$ that produced no viable pollen grains (Fig. 1).

In $\mathrm{F}_{2}$ hybrids, $\mathrm{PC} 1$ and $\mathrm{PC} 2$, which represent variation in plant overall size and leaf physiology, showed weak positive but significant correlations with PFR (Fig. 2). For individual traits, corolla length, stamen length, and leaf pigment concentration traits were significantly correlated with PFR (Additional file 1: Table S1).

\section{Mid-parent heterosis in 13 traits}

Mid-parent heterosis (MPH) was estimated as the percentage of the difference between the mean of $F_{1}$ or $F_{2}$ and the mid-parent mean divided by the mid-parent mean. We estimated MPH for PFR and 12 flower and leaf traits that we studied previously [41]. PFR displayed almost the lowest MPH among all of the traits (Table 1). $\mathrm{MPH}$ for PFR in $\mathrm{F}_{1}$ was $-44.5 \%$. The performance of 12 flower and leaf traits for $F_{1}$ hybrids, the $F_{2}$ population, and their parental lines is listed in our previously published paper [41]. In these traits, the concentration of 


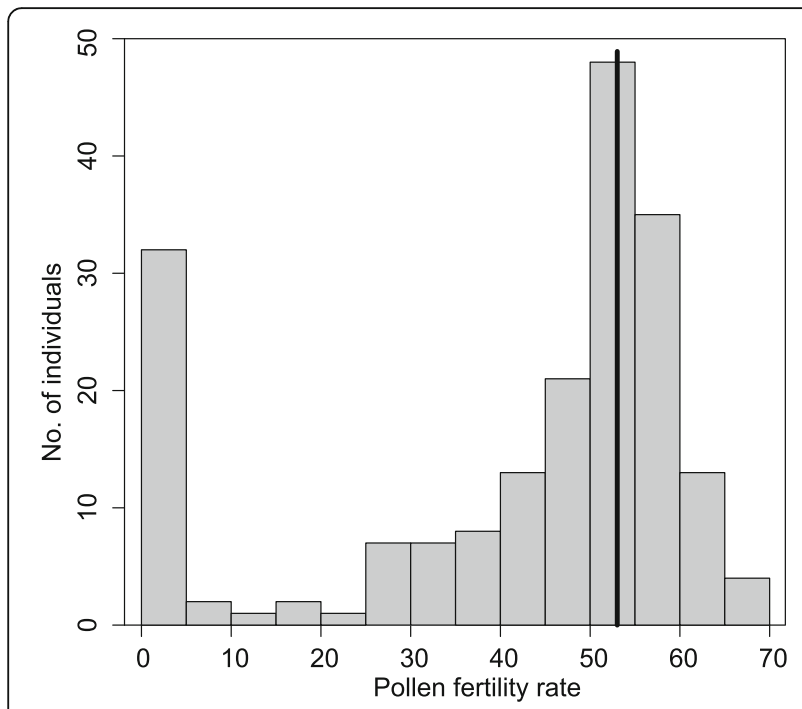

Fig. 1 Histogram of PFR in the $F_{2}$ population. Black vertical line indicates pollen fertility of $F_{1}$ hybrids leaf pigments showed the highest MPH (an average of $31.9 \%$ ) in $\mathrm{F}_{1}$ (Table 1).

Considerable transgressive segregation was observed for PFR and leaf pigments in $F_{2}$ individuals (Fig. 1; Table 1; data from [41]). As described above, all of the $\mathrm{F}_{2}$ individuals produced less fertile pollen than parental lines. Almost all of the $F_{2}$ individuals showed heterosis in leaf pigments (Table 1).

\section{QTL analyses}

The linkage map and QTL mapping analysis for floral and leaf traits were described in our previous study [41]. We detected QTL for PFR here with MapQTL v.6.0 software using $1 \mathrm{cM}$ increments [42]. Table 2 summarizes QTL mapping results for PFR, and Fig. 3 shows locations of QTLs. A total of eight QTLs were identified for PFR, which jointly explained $55.4 \%$ of the phenotypic variation in the $F_{2}$ population (Table 2). The phenotypic variation explained by each QTL ranged from $4.2 \%$ (PF02) to $17.8 \%$ (PF01). In particular, PF01 on LG02 is a major-effect locus for PFR, with an LOD value of 12.09. The $P$. depressa allele at PF01 resulted in $12.44 \%$ less pollen viability. Except PF01, all QTLs explained less than $7 \%$ of the total phenotypic variation (Fig. $4 \mathrm{a}$ and Table 2). These results suggest that the genetic
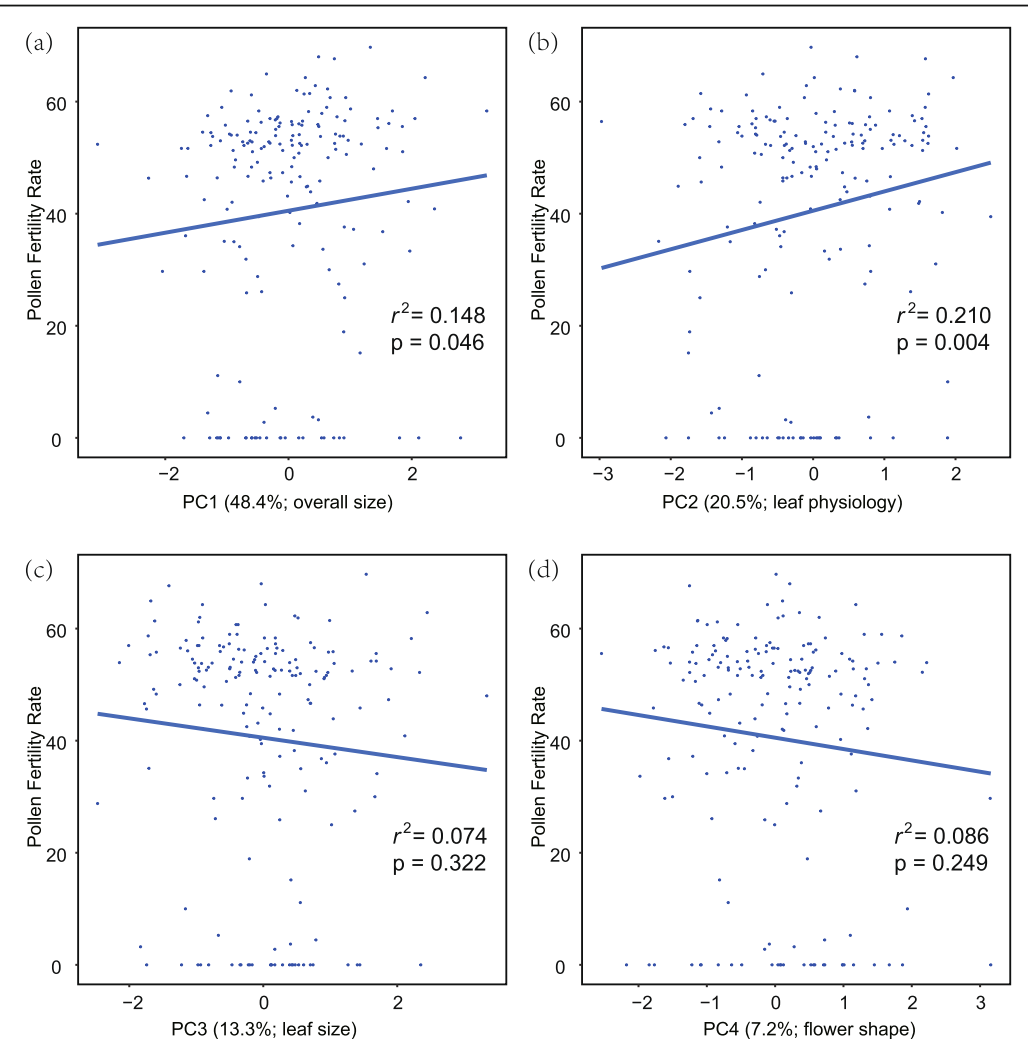

Fig. 2 Scatter plots showing the correlation between PFR and PCs derived from morphological and physiological traits. Percentages of total variation for each PC explained and interpretation for each PC represented were indicated in brackets 
Table 1 Mid-parent heterosis (MPH) for PFR and 12 flower and leaf traits in our previous study. If \%MPH $>0$, this represents the percentage of individuals showing $\mathrm{MPH}>0$ in all of the measured $\mathrm{F}_{2}$ individuals

\begin{tabular}{|c|c|c|c|c|c|c|}
\hline \multirow[t]{2}{*}{ Trait } & \multirow[t]{2}{*}{ Unit } & \multirow{2}{*}{$\begin{array}{l}\text { MPH } \\
\text { of } F_{1}\end{array}$} & \multicolumn{3}{|l|}{$\mathrm{MPH}$ of $\mathrm{F}_{2}$} & \multirow{2}{*}{$\begin{array}{l}\% \mathrm{MPH}> \\
0\end{array}$} \\
\hline & & & Mean \pm SD & Max & Min & \\
\hline Pollen fertility rate & $\%$ & $-44.5 \%$ & $-0.469 \pm 32.58$ & -0.047 & -1.000 & 0.00 \\
\hline Upper petal width & $\mathrm{mm}$ & $7.4 \%$ & $-0.103 \pm 13.21$ & 0.294 & -0.412 & 18.95 \\
\hline Lower petal width & $\mathrm{mm}$ & $1.2 \%$ & $-0.111 \pm 11.81$ & 0.231 & -0.438 & 20.53 \\
\hline Corolla width & $\mathrm{mm}$ & $5.0 \%$ & $-0.053 \pm 12.80$ & 0.479 & -0.339 & 32.63 \\
\hline Corolla height & $\mathrm{mm}$ & $18.6 \%$ & $-0.060 \pm 11.94$ & 0.330 & -0.407 & 35.26 \\
\hline Corolla length & $\mathrm{mm}$ & $-41.2 \%$ & $-0.007 \pm 13.94$ & 0.323 & -0.472 & 47.37 \\
\hline Pistil length & $\mathrm{mm}$ & $4.2 \%$ & $0.003 \pm 10.87$ & 0.284 & -0.245 & 50.00 \\
\hline Stamen length & $\mathrm{mm}$ & $-0.2 \%$ & $-0.002 \pm 10.63$ & 0.310 & -0.288 & 51.31 \\
\hline Maximum leaf length & $\mathrm{cm}$ & $-36.0 \%$ & $-0.389 \pm 24.89$ & 0.019 & -0.697 & 0.52 \\
\hline Maximum leaf width & $\mathrm{cm}$ & $-7.8 \%$ & $-0.120 \pm 25.91$ & 0.466 & -0.559 & 17.28 \\
\hline Chlorophyll a concentration & $\mathrm{mg} / \mathrm{m}^{2}$ & $36.4 \%$ & $0.559 \pm 11.75$ & 1.192 & -0.050 & 98.97 \\
\hline Chlorophyll b concentration & $\mathrm{mg} / \mathrm{m}^{2}$ & $35.1 \%$ & $0.484 \pm 19.23$ & 1.116 & -0.188 & 97.42 \\
\hline Carotenoid concentration & $\mathrm{mg} / \mathrm{m}^{2}$ & $24.2 \%$ & $0.496 \pm 11.90$ & 1.103 & -0.113 & 98.97 \\
\hline
\end{tabular}

architecture of pollen fertility contains a major-effect QTL plus numerous minor-effect QTLs.

For five of the eight pollen fertility QTLs (PF01, PF02, PF05, PF06, and PF07), P. danxiaensis alleles were associated with decreases in pollen viability (Table 2 and Figs. 4b and 5). Analysis of the dominance effect showed that three QTLs (PF02, PF03, and PF06) were dominant with $P$. danxiaensis alleles, and the other five were dominant with $P$. depressa alleles (Table 2 and Figs. 4c and 5).

Comparing with previous QTLs for 12 flower and leaf traits for the same population, we found two QTLs for pollen fertility (PF02 and PF04) overlapped with QTLs for floral morphology (corolla length and stamen length) (Table 2 and Fig. 3). We calculated the significance of correspondence between these overlapping QTLs and found that both pairs showed significantly more overlap than expected by chance $(P<0.05)$. In addition, we found two additional floral morphology QTLs (upper petal width and corolla width) were tightly linked (about 5 cM) with PF02 (Table 2 and Fig. 3). As we previously found that divergence in flower morphology was driven by natural selection [41], there was probably a hitchhiking effect between hybrid sterility and floral traits.

Table 2 Summary of QTLs detected for PFR and overlapping loci for two floral morphological traits

\begin{tabular}{|c|c|c|c|c|c|c|c|}
\hline Locus ID & LG & Position (cM) & Nearest RAD locus & LOD & 1.5 LOD unit of support (range in cM) & \% Expl. & QTL direction \\
\hline PF01 & 2 & 165.8 & 152,874 & 12.09 & $165.19-165.78$ & 17.8 & - \\
\hline PF02 & 6 & 102.4 & 170,253 & 3.18 & 101.00-103.36 & 4.2 & - \\
\hline PF03 & 7 & 77.6 & 19,822 & 5.11 & $76.63-78.69$ & 6.9 & + \\
\hline PF04 & 11 & 105.7 & 152,571 & 4.18 & 100.53-108.68 & 5.5 & + \\
\hline PF05 & 12 & 70.3 & 42,292 & 3.76 & $69.48-70.27$ & 5.0 & - \\
\hline PF06 & 13 & 114.2 & 117,627 & 4.67 & $113.51-114.19$ & 6.3 & - \\
\hline PF07 & 16 & 40.8 & 60,459 & 3.66 & $40.01-41.36$ & 4.9 & - \\
\hline PF08 & 18 & 27.4 & 114,119 & 3.63 & 21.30-29.35 & 4.8 & + \\
\hline SL03 & 6 & 103.362 & 33,528 & 4.34 & $102.362-105.998$ & 5.3 & + \\
\hline UPW06 & 6 & 107.81 & 94,126 & 5.9 & $106.95-107.81$ & 7.6 & + \\
\hline CW02 & 6 & 107.81 & 101,936 & 5.11 & $107.81-108.37$ & 7.7 & + \\
\hline CLO7 & 11 & 107.676 & 124,189 & 3.74 & 103.676-111.772 & 4.4 & + \\
\hline
\end{tabular}

LG refers to the linkage group with which the QTL was detected. QTL interval and position are given in centimorgan (cM). The LOD significance level was determined by 10,000 permutations. \% Expl. represents the phenotypic variation effect. In the QTL direction column, +/- indicates QTL effects are/are not in the direction of trait divergence between parental species. All of the QTLs were found at the $0.5 \%$ significance level

$P F$ pollen fertility, SL stamen length, UPW upper petal width, CW corolla width, $C L$ corolla length 


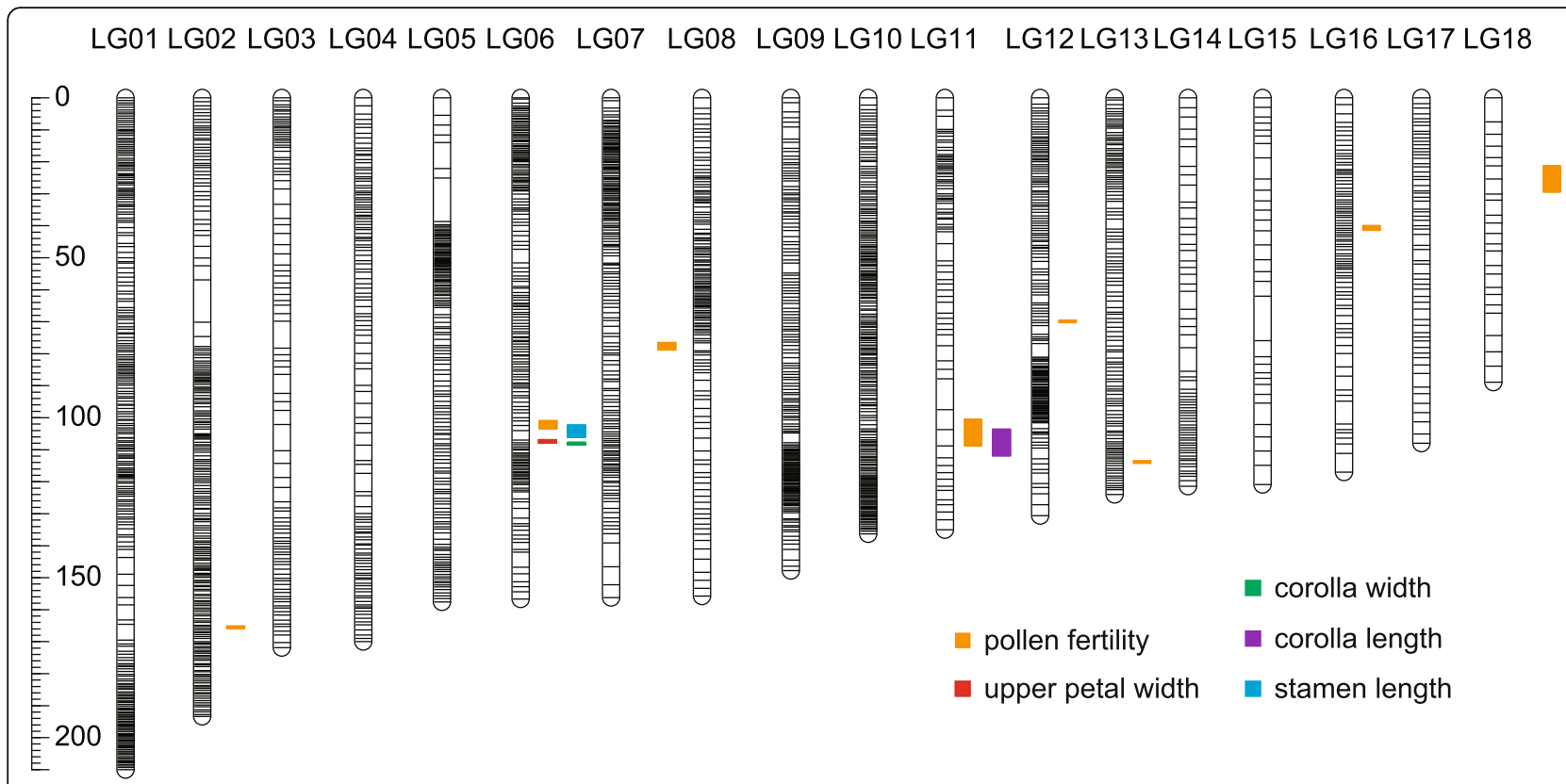

Fig. 3 Genome location of significant PFR QTLs and two co-localized floral morphology QTLs. Confidence intervals of QTLs are illustrated on the right side of linkage groups

However, we did not find the evidence of QTL overlap between PFR and leaf physiology.

\section{Gene action and locus interaction}

According to gene action estimation, two of the eight identified QTLs (PF01 and PF02) exhibited partial dominance, and the other six exhibited overdominance (Table 3). For the six overdominant loci, heterozygous genotypes reduced the pollen fertility at loci of PF03 and PF06 (Table 3 and Fig. 5). The underdominance evidenced the conflict between parental alleles. Conversely, the heterozygous genotype was favorable for pollen fertility at four loci (PF04, PF05, PF07, and PF08; Table 3 and Fig. 5). The overdominance suggests that heterosis occurs at some loci.

We characterized pairwise QTL interactions to summarize epistatic interactions at a threshold of $P<$ 0.05 (Additional file 2: Table S2). Digenic interactions were identified between two QTL pairs: PF02/PF08 and PF03/PF08 (Fig. 6; Tables 3; Additional file 2: Table S2). A high level of male sterility occurred in individuals that harbored alleles from different parents at PF03 and PF08 simultaneously (e.g., heterozygous alleles or homozygous $P$. danxiaensis alleles at PF03 and homozygous $P$. depressa alleles at PF08). A similar pattern was observed between PF02 and PF08; lower PFR occurred in individuals that were homozygous for $P$. danxiaensis alleles at PF08 and homozygous for $P$. depressa alleles or heterozygous for both parental alleles at PF02.

The estimation of locus segregation distortion revealed that peak LOD markers and their surrounding markers of
PF02 distorted to the male parent $(P<0.05)$, while PF05 distorted to the female parent (Tables 3; Additional file 3: Table S3). Peak LOD markers and many surrounding markers of PF04, PF07 and PF08 distorted to female and male parents, respectively, even though not significantly at peak LOD markers (Additional file 3: Table S3).

\section{Discussion}

Hybrid inviability and sterility

Studying mechanisms of reproductive isolation barriers is important for improving our understanding of the process of speciation [3, 5]. We identified multiple potential prezygotic reproductive isolation barriers that could contribute to the divergence between sympatric $P$. depressa and P. danxiaensis [41]. However, the incomplete pollinator divergence and the overlapping flowering time indicate that the prezygotic reproductive isolation might be insufficient for blocking the hybridization between $P$. depressa and $P$. danxiaensis. In this study, approximately one-sixth of the $F_{2}$ hybrids showed complete male sterility (Fig. 1), which indicates hybrid male sterility has contributed, at least partially, to reproductive isolation between these two species.

Hybrid inviability and sterility are two of the most common forms of postzygotic isolation in plants, because they prevent gene flow between lineages. In our case, we studied MPH for hybrid fertility and 12 flower and leaf traits to unravel the mechanism of postzygotic isolation between $P$. depressa and $P$. danxiaensis in sympatry. In $F_{1}$ hybrids, pollen fertility showed a greater underdominance than all of the flower and leaf traits 

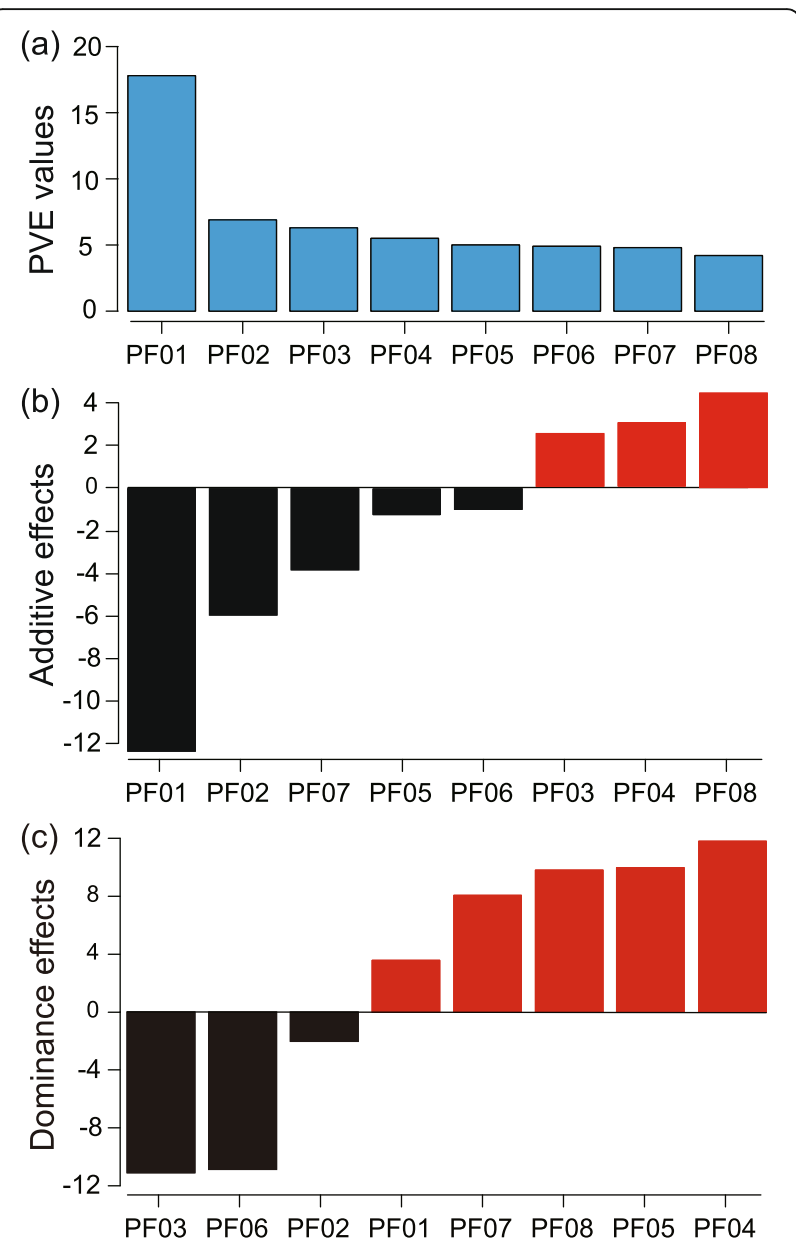

Fig. 4 The effect size, additive effect distribution, and dominance effect distribution of QTLs for hybrid PFR are shown in (a), (b), and (c). The $x$-axis indicates the name of each QTL. The $y$-axis in a indicates the phenotypic variation explained by each QTL (PVE). The $y$-axis in $\mathbf{b}$ and $\mathbf{c}$ indicates the additive and dominance effects of the $P$. depressa allele relative to the $P$. danxiaensis allele at each QTL

investigated (Table 1), suggesting that male sterility plays an important role in postzygotic reproductive isolation in this case. $F_{1}$ hybrids showed high concentrations of chlorophylls which represent potentially better photosynthesis capacities, but they did not show significant heterosis or depression for most of the traits for floral morphology and leaf size (abstract values of $\mathrm{MPH}$ were less than 10\%; Table 1). Considerable transgressive segregation of leaf pigments in $\mathrm{F}_{2}$ population indicated that the photosynthetic capacity of most $F_{2}$ hybrids was better than the parental lines (Table 1; data from [41]). This heterosis for photosynthesis was consistent with the action of loci in leaf pigments, 27 out of 30 loci were dominant or overdominant [41]. $\mathrm{MPH}$ value of all $\mathrm{F}_{2}$ individuals for PFR were negative, and this was possibly caused by genetic conflict (Table 1). Collectively, these results indicate hybrid sterility might play an important role in postzygotic reproductive isolation, while we did not observe apparent hybrid inviability, at least in our greenhouse. Further study of the relative importance of hybrid male sterility and hybrid inviability will be needed in the future.

Artificial crossing between $P$. depressa and $P$. danxiaensis produced vigorous but semi-sterile hybrids. Most Primulina species are narrow endemics with small population sizes [43]. Small-size populations usually have lower genetic diversity and are prone to accumulating deleterious recessive alleles [44-47]. If chlorophyll content and photosynthetic capacity are maladaptive in this system, it will make sense that genetic incompatibilities decrease fertility but increase vigor in interspecific hybrids here. Similar conclusions have also been drawn in many other plant species, such as Luffa [48] and rice [49-52].

\section{Genetic architecture of hybrid male sterility}

We found a polygenic basis for PFR in $P$. depressa $\times P$. danxiaensis $\mathrm{F}_{2}$ hybrids. We identified one major-effect QTL, which explained $17.8 \%$ of the hybrid population phenotypic variation. Seven minor-effect individual QTLs explained $4.2-6.9 \%$ of the phenotypic variance (Table 2). These QTLs jointly accounted for $55.4 \%$ of the total phenotypic variation in hybrid pollen fertility. Even though a low number of QTLs may have remained hidden due to the modest size of the mapping population $(N=195)$, the genetic architecture of postzygotic isolation in our system was polygenic, as we found a major-effect QTL plus numerous minor-effect QTLs.

The polygenic genetic basis of hybrid male fertility is consistent with that observed in other plant species. For example, eight-QTL hybrid sterility was identified between two closely related species of Solanum [53], and a number of different QTLs for male sterility were mapped in two subsequent mapping experiments $[54,55]$. Likewise, studies in Drosophila indicated that interspecific male and female sterility were highly polygenic (e.g., $[56,57])$. Tao et al. [22] identified a total of approximately 60 QTLs contributing to hybrid male sterility when Drosophila mauritiana is introgressed into a Drosophila simulans background, indicating that a polygenic genetic basis of hybrid male sterility is prevalent in both plants and animals. However, two-locus genetic incompatibility was reported to cause male sterility between species or subspecies in rice [58, 59] and Mimulus [15]. Lowry et al. [60] detected three sterility loci in hybrids of two Panicum hallii ecotypes. In general, fewer loci contribute to hybrid sterility in plants than in Drosophila, perhaps because plants largely lack sex chromosomes [56, 61].

In our Primulina study, the major-effect QTL contributes nearly $18 \%$ of the hybrid male sterility. This large effect makes it possible that divergence of this QTL is 

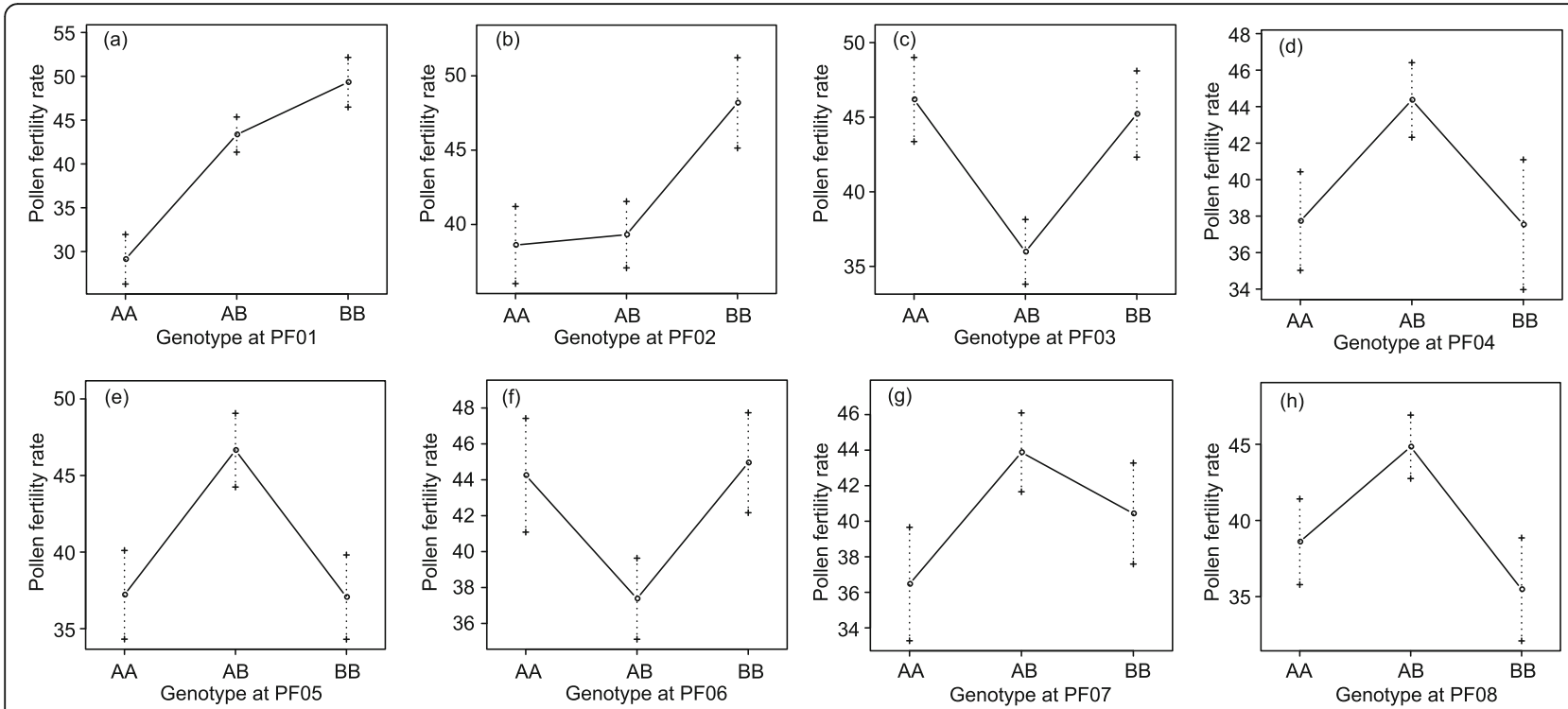

Fig. 5 Phenotypic trait means of different genotypes of each QTL

sufficient to impede gene flow and contribute to species integrity in the presence of vigorous hybrids. According to mean phenotype values at this major-effect locus (PF01), homologous P. danxiaensis alleles increase pollen fertility two-fold compared with homologous $P$. depressa alleles. Because mitochondrial genomes of hybrids were inherited maternally from $P$. danxiaensis, we suspect that CMS is a possible mechanism underlying hybrid male sterility here. Further elucidation of the molecular basis of PF01 will provide insight into its normal function within pure species.

The QTL overlap between PFR and flower morphology (corolla length and stamen length) suggests that hybrid male sterility is genetically correlated with floral morphological traits (Fig. 3 and data from [41]). In addition, a PFR QTL (PF02) was tightly linked to upper petal width and corolla width QTLs (Fig. 3 and data from [41]). This genotypic correlation pattern, including pleiotropic and tightly linked loci, was consistent with our phenotypic correlation analysis (Fig. 2; Additional file 1: Table S1). When a favorable mutation arises, the allele frequency of closely linked surrounding loci will also increase (i.e., genetic hitchhiking) [62]. Inversely, deleterious mutations will also eliminate the variation at their surrounding loci (i.e., background selection) [63]. In our case, flower morphology was divergently selected [41], and it may affect selection on hybrid male sterility genes when hitchhiking occurs. In consideration of both QTL overlap and close linkage of male sterility and morphological traits, we suspect that hybrid sterility evolved as an incidental by-product of hitchhiking. Groups of tightly linked minor-effect loci have larger selection

Table 3 The degree of dominance was calculated as dominance effect//additive effect|

\begin{tabular}{|c|c|c|c|c|c|c|c|c|c|c|}
\hline \multirow[t]{2}{*}{ Loci } & \multirow{2}{*}{$\begin{array}{l}\text { Additive } \\
\text { effect }\end{array}$} & \multirow{2}{*}{$\begin{array}{l}\text { Dominance } \\
\text { effect }\end{array}$} & \multirow{2}{*}{$\begin{array}{l}\text { Degree of } \\
\text { dominance }\end{array}$} & \multirow[t]{2}{*}{ GA } & \multirow{2}{*}{$\begin{array}{l}\text { Mean AA } \\
\text { phenotype }\end{array}$} & \multirow{2}{*}{$\begin{array}{l}\text { Mean } A B \\
\text { phenotype }\end{array}$} & \multirow{2}{*}{$\begin{array}{l}\text { Mean BB } \\
\text { phenotype }\end{array}$} & \multirow{2}{*}{$\begin{array}{l}\text { Epistatic } \\
\text { interaction } \\
\text { loci }\end{array}$} & \multicolumn{2}{|c|}{ Segregation distortion } \\
\hline & & & & & & & & & $P$-value & Significance \\
\hline PF01 & -12.44 & 3.58 & 0.29 & PD & 20.58 & 36.61 & 45.47 & & 0.802 & - \\
\hline PF02 & -5.98 & -1.99 & -0.33 & PD & 27.05 & 31.03 & 39.00 & PF08 & 0.019 & * \\
\hline PF03 & 2.48 & -11.06 & -4.46 & OD & 35.47 & 22.22 & 30.58 & PF08 & 0.355 & - \\
\hline PF04 & 3.00 & 11.80 & 3.93 & OD & 35.07 & 42.84 & 30.99 & & 0.089 & - \\
\hline PF05 & -1.21 & 9.97 & 8.22 & OD & 31.81 & 43.00 & 34.24 & & 0.013 & * \\
\hline PF06 & -0.95 & -10.83 & -11.34 & OD & 32.07 & 22.20 & 33.98 & & 0.087 & - \\
\hline PF07 & -3.83 & 8.06 & 2.10 & OD & 29.20 & 41.09 & 36.86 & & 0.447 & - \\
\hline PF08 & 4.46 & 9.80 & 2.20 & OD & 35.94 & 41.06 & 30.11 & PF02; PF03 & 0.439 & - \\
\hline
\end{tabular}

GA shows gene action modes, which are classified as follows: $A$, additive $(|d / a| \leq 0.20) ; P D$, partial dominance $(0.20<|d / a| \leq 0.80) ; D$, dominance $(0.80<|d / a| \leq 1.20) ;$ and $\mathrm{OD}$, overdominance $(|\mathrm{d} / \mathrm{a}|>1.20)$. Mean phenotype traits of the $P$. depressa homozygote $(\mathrm{AA})$, the heterozygote $(\mathrm{AB})$, and the $P$. danxiaensis homozygote $(\mathrm{BB})$ are shown. The $P$-value and significance of each locus segregation distortion were calculated * $P<0.05$ 

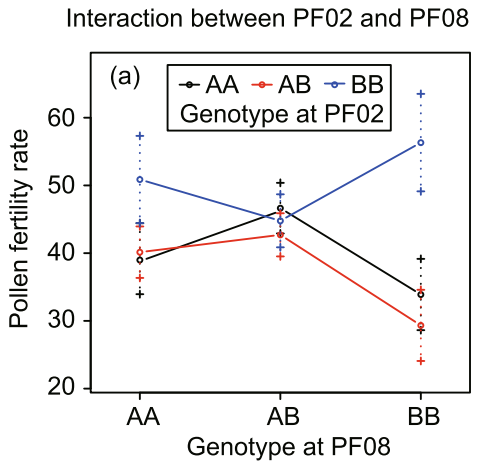

Interaction between PF03 and PF08

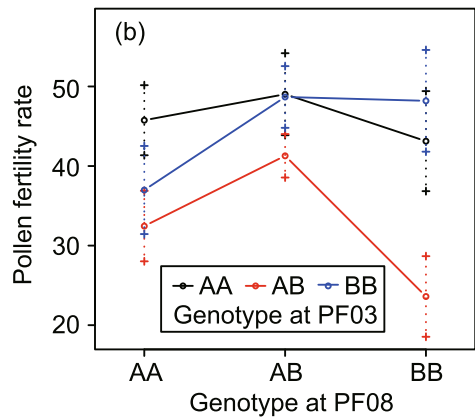

Fig. 6 Digenic interactions between two pairs of QTLs. AA, BB, and AB represent homozygous genotypes of male and female parents and a heterozygous genotype

coefficients than single loci with small phenotypic effects [25]. Therefore, a single locus that affects both adaptive and reproductive isolation traits would be the most probable way for selection to maintain differentially adapted species, even in the face of gene flow. These results are consistent with recent studies on Mimulus (e.g. $[28,29,64])$.

The coincidental (phenotypic and genotypic) correlation between PFR and flower morphology suggests developmental integration. In accordance with our results, a recent study in Mimulus found a genetic correlation between flower size and hybrid male fertility [35]. The inconsistent directionality of QTL effects suggests that reduced flower size in hybrids increases male fertility. In consideration of the association between selffertilization and reduced flower size [64], small flowers theoretically suppressed gene exchange between $P$. depressa and $P$. danxiaensis yet promote hybrid male fertility. However, further crossing experiments for estimating outcrossing rates are necessary to evidence the correlation between gene exchanging rate, flower size, and hybrid fertility.

Leaf pigments play important roles in responses to biotic and abiotic stresses and thus are sensitive to environmental changes [65-67]. Pollen fertility is easily affected by environmental factors as well $[18,19,68]$. Drought stress, a common instance of environmental stress, was reported to decrease both pollen fertility [69] and leaf chlorophyll content $[70,71]$ in wheat. This is one of the probable reason why, in our study, pollen fertility was correlated with leaf pigments phenotypically but not genotypically. Further work is needed to test the relationships among stresses, leaf pigments and pollen fertility.

\section{Complexity of hybrid sterility}

Significant negative epistatic interactions were observed between two pairs of QTLs: PF02/PF08 and PF03/PF08 (Fig. 6; Table 3; Additional file 2: Table S2). This indicates that the
BDM model involving three loci contributes to hybrid male sterility. According to the BDM model, sterility is more polymorphic within plant species than animal species (reviewed in [61]). The polygenic BDM model in our case implies the sterility may be under complex genetic control.

Besides the BDM model, segregation distortion is very common at loci surrounding hybrid male fertility QTLs in our study. Two QTLs (PF02 and PF05) showed significant segregation distortion at their peak LOD and surrounding loci (Additional file 3: Table S3). PF04, PF07, and PF08 did not show significant segregation distortion at peak LOD loci, but their surrounding loci did (Additional file 3: Table S3). The segregation distortion may resulted from gametes abortion (prezygotic selection) or the selective fertilization of particular genotypes (postzygotic selection). Unfortunately, we couldn't distinguish them from a single $F_{2}$ linkage map (reviewed by [72, 73]). Correlations between locus segregation distortion and male sterility has been reported in a few systems, including Drosophila (e.g., [74-76]). In rice, segregation distortion sometimes has significant effects on hybrid male sterility [77-79]. To our knowledge, however, this is the first study on correlation between locus segregation distortion and hybrid male fertility in non-model plants.

In contrast to Mimulus species [15], we here show a complex genetic basis for hybrid male sterility between $P$. depressa and $P$. danxiaensis. Hybrid male sterility between Mimulus guttatus and Mimulus nasutus were almost results from a simple genetic incompatibility between a single pair of heterospecific loci. In contrast, our results show a complex genetic basis of hybrid male sterility, including a BDM model involving three loci and genetic correlations with flower morphology. Further genetic dissection may reveal that more complicated mechanisms underling each locus. A more complex genetic architecture of a reproductive isolation barrier will reduce the rate of gene flow across the entire genome to a greater extent than a barrier governed by only a few 
major loci [6]. Therefore, the identified complex genetic architecture of hybrid male sterility might make a critical contribution to the maintainance of species identity between $P$. depressa and $P$. danxiaensis.

\section{Conclusions}

In this study, we found that hybrid male sterility in Primulina is controlled by a polygenic genetic basis. The genetic incompatibility involves a three-locus BDM model, even though the single largest-effect locus is not involved in. Hybrid male sterility is genetically correlated with floral morphology and divergence hitchhiking may have occurred between them. This complex pattern is in contrast to the simple genetic incompatibility model found in Mimulus [15]. The identified complex genetic architecture of hybrid male sterility might play an important role in maintaining species identity between $P$. depressa and $P$. danxiaensis. Continued studies of reciprocal backcross can provide detailed incompatibility patterns and further our understanding of evolutionary forces driving postzygotic reproductive isolation in this system.

\section{Methods}

\section{Plant materials and growth conditions}

We collected parental lines (P. depressa and P. danxiaensis) at the sympatric site of Danxia Mountain, Guangdong, South China. With the permission of Danxia Mountain National Park Commission, all samples were originally collected following methods that met the guidelines of Regulations on Wild Plants Protection (People's Republic of China) for the use of plants in research. Voucher specimens of P. depressa (DXSO2) and P. danxiaensis (DXS04) were deposited in the South China Botanical Garden Herbarium (IBSC). We created a $\mathrm{F}_{2}$ mapping population, including 201 individuals, by self-fertilizing one $F_{1}$ plant derived from a cross between an individual of $P$. depressa ( $\delta$ ) and an individual of $P$. danxiaensis ( $\left(\right.$ ). $\mathrm{F}_{1}$ hybrids, male and female parents, and $201 \mathrm{~F}_{2}$ individuals (the mapping population) were genotyped by restriction site-associated DNA sequencing (RAD-seq). A high-density linkage map was constructed with 2484 markers that were spaced across 18 linkage groups. For further details, see [41].

To determine male fertility, we planted parental populations, $F_{1}$ hybrids, and the $F_{2}$ population in a greenhouse in South China Botanical Garden under fluorescent light to provide a 14-h day length, with a temperature of $\sim 26^{\circ} \mathrm{C}$. Plants were watered by subirrigation as needed and fertilized weekly.

\section{Male fertility assessment and pollen staining}

Pollen viability was assessed by staining pollens with TTC [80]. For pollen staining, we collected the first three flowers per individual on the day the flower opened. These flowers contained mature pollen, and there was no bias in the analysis of each individual. We halved anthers with a pair of forceps, and then pollen was squeezed into $50 \mu \mathrm{l}$ of TTC ( $1 \% \mathrm{w} / \mathrm{v}$ in $50 \%$ sucrose) solution on a glass slide and incubated in the dark at room temperature for $15 \mathrm{~min}$. TTC white compound can be enzymatically converted to red 1,3,5-triphenylformazan (TPF) by various dehydrogenases in metabolically active pollen. Therefore, red stained pollen grains were considered viable, while faintly stained or empty pollen were non-viable. More than 100 pollen grains were observed for each flower under the microscope. The percentage of viable pollens in the total examined pollens was calculated as the pollen fertility rate (PFR).

\section{Phenotypic data analysis}

We previously performed principal component analysis (PCA) on seven floral traits (upper petal width, lower petal width, corolla width, corolla height, corolla length, pistil length and stamen length), three leaf physiological traits (concentrations of chlorophyll a, chlorophyll b, and carotenoids), and two morphological traits (leaf length and leaf width). The first two principal components (PCs) accounted for 48.4 and $20.5 \%$ of the total measured phenotypic variation, with the first component correlating with overall size (in particular the size of floral traits) and the second with leaf physiology traits. For detail information, see [41]. Here, we calculated Pearson's correlation coefficients between PFR and the 12 previously studied traits and PCs derived from them.

In order to evaluate the hybrid phenotypic performances, a previously developed mid-parent heterosis $(\mathrm{MPH})$ statistical test $[81,82]$ was used. MPH was calculated using the following formula:

$$
\mathrm{MPH}=\frac{\text { Hybrid mean }- \text { Mid-parent mean }}{\text { Mid-parent mean }} \times 100 \%,
$$

Where mid-parent mean represents the average of both parents with respect to a trait of interest. In this study, we performed MPH analysis on 13 phenotypic traits mentioned above.

\section{QTL mapping}

The linkage map used in this study was a highly resolved RAD-seq-based SNP map with 2484 markers distributed across the 18 linkage groups with an average distance of $0.96 \mathrm{cM}$ [41]. The QTL intervals of PFR were defined on the linkage map with MapQTL v.6.0 software [42]. Before QTL mapping, a genome-wide and chromosome-level LOD score threshold $(P<0.05)$ for declaring the presence of QTLs was determined using the permutation test (10, 000 replications). Based on the permutation results, an LOD score threshold of 3.0 was set for the trait to declare 
the presence of a significant QTL. The potential QTLs were initially detected employing the Interval Mapping (IM) algorithm. Then, markers with the highest LOD values were selected as cofactors, and the final set of markers detected at $P<0.05$ after automatic cofactor selection were further included in the Multiple QTL Model (MQM). Cofactor selection and MQM analysis were repeated until the best possible set of QTLs was found, and then each QTL was characterized by its maximal LOD score. The 1.5-LOD support intervals were estimated in centimorgans (cM) all of the significant QTLs detected. Final QTL maps were drawn with the help of the graphical package MapChart v2.2 [83].

To evaluate the significance of correspondence between QTL and PFR or other traits that we studied previously [41], we calculated the probability of obtaining the observed number of matching QTLs by chance alone [84]. This probability was estimated using a hypergeometric probability distribution function [85]. The equation used is shown in [56].

\section{Gene action and locus interaction analysis}

Gene action was proposed to estimate the relative importance of the dominance effect and the additive effect, and it was estimated by calculating $|\mathrm{d} / \mathrm{a}|=\mid \mathrm{dom}$ inance effects/additive effects| [86]. Results were defined as follows: A, additive $(|\mathrm{d} / \mathrm{a}| \leq 0.20)$; $\mathrm{PD}$, partial dominance $(0.20<|\mathrm{d} / \mathrm{a}| \leq 0.80) ; \mathrm{D}$, dominance $(0.80<|\mathrm{d} / \mathrm{a}| \leq$ 1.20); and OD, overdominance $(|\mathrm{d} / \mathrm{a}|>1.20)$. Thresholds were firstly proposed by [86], and commonly used in many following studies (e.g., [87-89]).

Digenic epistatic interactions were analyzed with markers closer to QTL peaks using R/qtl [90]. The proportion of variance explained by epistasis was tested by comparing the residual of the full model containing all of the single-locus effects and two-locus interaction effects with that of the reduced model containing all of the single-locus effects but excluding two-locus interaction effects.

In addition to gene action and digenic interactions, segregation distortion of mapped QTLs and their closely linked markers was estimated. The degree of marker segregation distortion in the $F_{2}$ generation was determined by marker data comparison against the expected 1:2:1 ratio using the Chi square test, where significant distortion was declared at $P<0.05$.

\section{Supplementary information}

Supplementary information accompanies this paper at https://doi.org/10. 1186/s12862-020-01617-4

Additional file 1 : Table S1. Linear correlation (Pearson) among the previously studied 12 flower and leaf traits and pollen fertility rate in $F_{2}$ population. Values in bold are significant $(p<0.05)$.
Additional file $\mathbf{2}$ : Table S2. Summary of significant epistatic interactions between QTLs. Epistasis analyses among the identified QTLs were conducted with R/qtl. Var (\%), F-value, and $P$-value represent percentage of variance explained, $F$ statistics, and $F$ distribution, respectively. ${ }^{*} P<0.05$, ${ }^{* *} P<0.01$.

Additional file $\mathbf{3}$ : Table S3. Genotype distribution of peak LOD loci of QTLs and their surrounding ten loci. The Chi square test was used. ${ }^{*} P<$ $0.05{ }^{* *} P<0.01$. Peak LOD loci of each QTL are presented in bold.

\section{Abbreviations}

BDM: Bateson-Dobzhansky-Muller; CMS: Cytoplasmic male sterility; PFR: Pollen fertility rate; QTL: Quantitative trait loci; RAD: Restriction siteassociated DNA

\section{Acknowledgements}

We highly acknowledge Juan Liu for her support in the samples collection.

\section{Authors' contributions}

This study was conceived by MK and CF. Collection and identification of field materials were performed by LY and MK. Sample preparation and nuclei isolation were performed by CF and HY. Data analysis was conducted by CF and HY. CF and MK wrote the paper. All authors read and approved the final manuscript.

\section{Funding}

This work was supported by the Natural Science Foundation of China (31570338) and the Strategic Priority Research Program of Chinese Academy of Sciences (XDB31000000) to MK. CF received support from the China Postdoctoral Science Foundation (2019 M653111). The funder had no role in the study design, data collection and analysis, interpretation of data, or writing of the manuscript.

\section{Availability of data and materials}

Pollen fertility rate data for QTL analysis are available at the FIGSHARE repository: DOI: https://doi.org/10.6084/m9.figshare.8949218.

Ethics approval and consent to participate

Not applicable.

Consent for publication

Not applicable.

Competing interests

The authors declare that they have no competing interests.

\section{Author details}

${ }^{1}$ Key Laboratory of Plant Resources Conservation and Sustainable Utilization, South China Botanical Garden, Chinese Academy of Sciences, Guangzhou 510650, China. ${ }^{2}$ Center of Conservation Biology, Core Botanical Gardens, Chinese Academy of Sciences, Guangzhou 510650, China. ${ }^{3}$ University of Chinese Academy of Sciences, Beijing 100049, China.

Received: 19 July 2019 Accepted: 21 April 2020

Published online: 29 April 2020

\section{References}

1. Dobzhansky TG. Genetics and the origin of species. New York: Columbia University Press; 1937.

2. Grant V. Plant speciation. New York: Columbia University. Press; 1981.

3. Coyne JA, Orr HA. Speciation. Sunderland: Sinauer Associates; 2004.

4. Ramsey J, Bradshaw HD Jr, Schemske DW. Components of reproductive isolation between the monkeyflowers Mimulus lewisii and M. cardinalis (Phrymaceae). Evolution. 2003;57:1520-34.

5. Nosil P, Vines TH, Funk DJ. Perspective: reproductive isolation caused by natural selection against immigrants from divergent habitats. Evolution. 2005:59:705-19.

6. Lowry DB, Modliszewski JL, Wright KM, et al. The strength and genetic basis of reproductive isolating barriers in flowering plants. Philos T R Soc B. 2008; 363:3009-21. 
7. Orr H, Presgraves DC. Speciation by postzygotic isolation: forces, genes and molecules. Bioessays. 2000;22:1085-94.

8. Long Y, Zhao L, Niu B, et al. Hybrid male sterility in rice controlled by interaction between divergent alleles of two adjacent genes. Proc Natl Acad Sci U S A. 2008;105:18871-6.

9. Hinchliffe DJ, Turley RB, Naoumkina M, et al. A combined functional and structural genomics approach identified an EST-SSR marker with complete linkage to the Ligon lintless-2 genetic locus in cotton (Gossypium hirsutum L.). BMC Genomics. 2011;12:445.

10. Orr H. The population genetics of speciation: the evolution of hybrid incompatibilities. Genetics. 1995;139:1805-13.

11. Moyle LC, Olson MS, Tiffin P. Patterns of reproductive isolation in three angiosperm genera. Evolution. 2004;58:1195-208.

12. Scopece G, Widmer A, Cozzolino S. Evolution of postzygotic reproductive isolation in a guild of deceptive orchids. Am Nat. 2008:171:315-26.

13. Martin $H$, Touzet $P$, Dufay $M$, et al. Lineages of Silene nutans developed rapid, strong, asymmetric postzygotic reproductive isolation in allopatry. Evolution. 2017;71:1519-31.

14. Fishman L, Sweigart AL. When two rights make a wrong: the evolutionary genetics of plant hybrid incompatibilities. Annu Rev Plant Biol. 2018;69:707-31.

15. Sweigart A, Fishman L, Willis J. A simple genetic incompatibility causes hybrid male sterility in Mimulus. Genetics. 2006;172:2465-79.

16. Luo $\mathrm{D}, \mathrm{Xu} \mathrm{H}$, Liu Z, et al. A detrimental mitochondrial-nuclear interaction causes cytoplasmic male sterility in rice. Nat Genet. 2013;45:573-7.

17. Simon M, Durand S, Pluta N, et al. Genomic conflicts that cause pollen mortality and raise reproductive barriers in Arabidopsis thaliana. Genetics. 2016:203:1353-67.

18. Chen L, Liu Y. Male sterility and fertility restoration in crops. Annu Rev Plant Biol. 2014;65:579-606.

19. Yang Z, Zhang ZL, Zhang $T$, et al. The effect of season-long temperature increases on rice cultivars grown in the central and southern regions of China. Front Plant Sci. 2017;8:1908.

20. Oka H. Analysis of genes controlling $F_{1}$ sterility in rice by the use of isogenic lines. Genetics. 1974;77:521-34.

21. Ikehashi $H$, Araki $H$. Genetics of $F_{1}$ sterility in remote crosses of rice (Oryza sativa L.). In: Rice genetics I; 1986. p. 119-30

22. Tao Y, Zeng ZB, Li J, et al. Genetic dissection of hybrid incompatibilities between Drosophila simulans and D. mauritiana. II. Mapping hybrid male sterility loci on the third chromosome. Genetics. 2003;164:1399-418.

23. Morán T, Fontdevila A. Genome-wide dissection of hybrid sterility in Drosophila confirms a polygenic threshold architecture. J Hered. 2014;105:381-96.

24. Davies $B$, Hatton E, Altemose N, et al. Re-engineering the zinc fingers of PRDM9 reverses hybrid sterility in mice. Nature. 2016;530:171-6.

25. Ekblom R, Galindo J. Applications of next generation sequencing in molecular ecology of non-model organisms. Heredity. 2011;107:1-15.

26. Yeaman S, Otto SP. Establishment and maintenance of adaptive genetic divergence under migration, selection, and drift. Evolution. 2011;65:2123-9.

27. Yeaman $S$, Whitlock MC. The genetic architecture of adaptation under migration-selection balance. Evolution. 2011;65:1897-911.

28. Wright KM, Lloyd D, Lowry DB, et al. Indirect evolution of hybrid lethality due to linkage with selected locus in Mimulus guttatus. PLoS Biol. 2013;11: e1001497.

29. Ferris KG, Barnett $L L$, Blackman BK, et al. The genetic architecture of local adaptation and reproductive isolation in sympatry within the Mimulus guttatus species complex. Mol Ecol. 2017;26:208-24.

30. Feder JL, Egan SP, Nosil P. The genomics of speciation-with-gene-flow. Trends Genet. 2012;28:342-50.

31. Feder JL, Gejji R, Yeaman S, et al. Establishment of new mutations under divergence and genome hitchhiking. Philos T R Soc B. 2012:367:461-74.

32. Via S. Divergence hitchhiking and the spread of genomic isolation during ecological speciation-with-gene-flow. Philos T R Soc B. 2012;367:451-60.

33. Chase CD. Cytoplasmic male sterility: a window to the world of plant mitochondrial-nuclear interactions. Trends Genet. 2007;23:81-90.

34. Barr CM, Fishman L. Cytoplasmic male sterility in Mimulus hybrids has pleiotropic effects on corolla and pistil traits. Heredity. 2011;106:886-93.

35. Fishman L, Beardsley PM, Stathons A, et al. The genetic architecture of traits associated with the evolution of self-pollination in Mimulus. New Phytol. 2015;205:907-17.

36. Wright KM, Loyd D, Lowry DB, et al. Indirect evolution of hybrid lethality due to linkage with a selected locus in Mimulus quttatus. PLoS Biol. 2013;11: e1001497.
37. Hooker JD. Chirita depressa Hook. f. Curtis's botanical magazine; 1892. p. 118:t.213.

38. Shen RJ, Lin SS, Yu Y, et al. Chiritopsis danxiaensis sp. nov. (Gesneriaceae) from Mount Danxiashan, South China. Nord J Bot. 2010;28:728-32.

39. Weber A, Clark JL, Möller M. A new formal classification of Gesneriaceae. Selbyana. 2013;31:68-94.

40. Kong $\mathrm{H}$, Condamine $\mathrm{FL}$, Harris AJ, et al. Both temperature fluctuations and East Asian monsoons have driven plant diversification in the karst ecosystems from southern China. Mol Ecol. 2017;26:6414-29.

41. Feng C, Feng C, Yang $L$, et al. Genetic architecture of quantitative flower and leaf traits in a pair of sympatric sister species of Primulina. Heredity. 2019;122:864-76.

42. van Ooijen JW. MapQTL 5 software for the mapping of quatitative trait loci in experimental populations. Wageningen: Kyazma, BV; 2004.

43. Wei YG. Gesneriaceae of South China. Nanning: Guangxi Science and Technology Publishing House; 2010.

44. Bomblies K, Weigel D. Hybrid necrosis: autoimmunity as a potential geneflow barrier in plant species. Nat Rev Genet. 2007;8:382-93.

45. Charlesworth D, Willis JH. The genetics of inbreeding depression. Nat Rev Genet. 2009;10:783-96.

46. Honnay $\mathrm{O}$, Jacquemyn $\mathrm{H}$. Susceptibility of common and rare plant species to the genetic consequences of habitat fragmentation. Conserv Biol. 2007; 21:823-31.

47. Vranckx GUY, Jacquemyn $H$, Muys B, et al. Meta-analysis of susceptibility of woody plants to loss of genetic diversity through habitat fragmentation. Conserv Biol. 2011;26:228-37.

48. Wu H, He X, Gong H, et al. Genetic linkage map construction and QTL analysis of two interspecific reproductive isolation traits in sponge gourd. Front Plant Sci. 2016. https://doi.org/10.3389/fpls.2016.00980.

49. Widmer A, Lexer C, Cozzolino S. Evolution of reproductive isolation in plants. Heredity. 2009;102:31-8.

50. Ouyang YD, Liu YG, Zhang QF. Hybrid sterility in plant: stories from rice. Curr Opin Plant Biol. 2010;13:186-92.

51. Huang JZ, E ZG, Zhang HL, et al. Workable male sterility systems for hybrid rice: genetics, biochemistry, molecular biology, and utilization. Rice. 2014;7:13.

52. Xie $Y$, Niu B, Long $Y$, et al. Suppression or knockout of SaF/SaM overcomes the Sa-mediated hybrid male sterility in rice. J Integr Plant Biol. 2017;59:669-79.

53. Moyle LC, Graham EB. Genetics of hybrid incompatibility between Lycopersicon esculentum and L. hirsutum. Genetics. 2005;169:355-73.

54. Moyle LC, Nakazato T. Hybrid incompatibility "snowballs" between Solanum species. Science. 2010;329:1521-3.

55. Moyle LC, Nakazato T. Comparative genetics of hybrid incompatibility: sterility in two Solanum species crosses. Genetics. 2008;179:1437.

56. Wu C-I, Davis AW. Evolution of postmating reproductive isolation-the composite nature of Haldane's rule and its genetic bases. Am Nat. 1993;142: $187-212$.

57. Davis AW, Wu C-I. The broom of the sorcerer's apprentice: the fine structure of a chromosomal region causing reproductive isolation between two sibling species of Drosophila. Genetics. 1996;143:1287-98.

58. Sano Y. The genic nature of gamete eliminator in rice. Genetics. 1990;125: 183-91.

59. Kubo T, Yoshimura A. Epistasis underlying female sterility detected in hybrid breakdown in a Japonica-Indica cross of rice (Oryza sativa L.). Theor Appl Genet. 2005;110:346-55.

60. Lowry DB, Hernandez K, Taylor SH, et al. The genetics of divergence and reproductive isolation between ecotypes of Panicum hallii. New Phytol. 2015;205:402-14.

61. Rieseberg LH, Willis JH. Plant speciation. Science. 2007;317:910-4.

62. Maynard S, Haigh J. The hitch-hiking effect of a favourable gene. Genet Res. 1974;23:23-35.

63. Stephan W. Genetic hitchhiking versus background selection: the controversy and its implications. Philos Trans R Soc B. 2010;365:1245-53.

64. Sicard A, Lenhard $\mathrm{M}$. The selfing syndrome: a model for studying the genetic and evolutionary basis of morphological adaptation in plants. Ann Bot. 2011;107:1433-43

65. Smth $\mathrm{H}$, Holmes MG. The function of phytochrome in the natural environment-III. Measurement and calculation of phytochrome photoequilibria. Photochem Photobiol. 1977;25:547-50.

66. Guanter L, Zhang Y, Jung M, et al. Global and time-resolved monitoring of crop photosynthesis with chlorophyll fluorescence. Proc Natl Acad Sci U S A. 2014;111:E1327-33. 
67. Toscano S, Trivellini A, Ferrante A, et al. Physiological mechanisms for delaying the leaf yellowing of potted geranium plants. Sci Hortic. 2018;242: 146-54.

68. Pucher A, Hash CT, Wallace JG, et al. Mapping a male-fertility restoration locus for the A 4 cytoplasmic-genic male-sterility system in pearl millet using a genotyping-by-sequencing-based linkage map. BMC Plant Biol. 2018;18:65.

69. Ji X, Shiran B, Wan J, et al. Importance of pre-anthesis anther sink strength for maintenance of grain number during reproductive stage water stress in wheat. Plant Cell Environ. 2010;33:926-42.

70. Pradhan GP, Prasad PW, Fritz AK, et al. Effects of drought and high temperature stress on synthetic hexaploid wheat. Funct Plant Biol. 2012;39: 190-8.

71. Hussain S, Chachar Q, Keerio Ml, et al. Physiological and biochemical response of wheat genotypes under temperature stress. Pak J Bot. 2020;52: 365-74.

72. Li X, Wang X, Brummer E. Prevalence of segregation distortion in dioploid alfalfa and its implications for genetics and breeding applications. Theor Appl Genet. 2011;123:667-79.

73. Chancerel E, Lamy J, Lesur I, et al. High-density linkage mapping in a pine tree reveals a genomic region associated with inbreeding depression and provides clues to the extent and distribution of meiotic recombination. BMC Biol. 2013;11:50

74. Orr H, Irving S. Segregation distortion in hybrids between the Bogota and USA subspecies of Drosophila pseudoobscura. Genetics. 2005;169:671-82.

75. Phadnis N, Orr HA. A single gene causes both male sterility and segregation distortion in Drosophila hybrids. Science. 2009;323:376-9.

76. Phadnis N. Genetic architecture of male sterility and segregation distortion in Drosophila pseudoobscura Bogota-USA hybrids. Genetics. 2011;111:132324.

77. Koide $Y$, Ikenaga M, Sawamura N, et al. The evolution of sex independent transmission ratio distortion involving multiple allelic interactions at a single locus in rice. Genetics. 2008;180:409-20.

78. Yang J, Zhao X, Ke C, et al. A kille-protector system regulates both hybrid sterility and segregation distortion in rice. Sciences. 2012;337:1336-40.

79. Shan J, Cai Z, Zhang Y, et al. The underlying pathway involved in intersubspecific hybrid male sterility in rice. Genomics. 2019;111:1447-55.

80. Stanley R, Linskens H. Pollen-biology, biochemistry, management. Berlin, Heidelberg and New York: Springer-Veriag; 1974

81. Comstock RE, Robinson HF. "Estimation of average dominance of genes." in: Heterosis, edited by JW Gowen. lowa: lowa State College Press; 1952. pp. 494-516.

82. Melchinger AE, Utz HF, Piepho HP, et al. The role of epistasis in the manifestation of heterosis: a systems-oriented approach. Genetics. 2007;177: $1815-25$.

83. Huang X, Yang S, Gong J, et al. Genomic analysis of hybrid rice varieties reveals numerous superior alleles that contribute to heterosis. Nat Commun. 2015;6:6258. Voorrips R. MapChart: software for the graphical presentation of linkage maps and QTLs. J Hered. 2002;93:77-8.

84. Lin YR, Schertz KF, Paterson AH. Comparative analysis of QTLs affecting plant height and maturity corss the Poaceae, in reference to an interspecific sorghum population. Genetics. 1995;14:391-411.

85. Larsen R, Marx M. An introduction to probability and its applications. Englewood Cliffs: Prentice Hall Inc; 1985.

86. Edwards MD, Stuber CW, Wendel JF. Molecular-marker-facilitated investigations of quantitative-trait loci in maize. I. Numbers, genomic distribution and types of gene action. Genetics. 1987;116:113-25.

87. Stuber C, Edwards M, Wendel J. Molecular marker facilitated investigation of quantitative trait loci in maize. II. Factors influencing yields and its component traits. Crop Sci. 1987:27:639-48.

88. Jiang $L, G e M, Z$ hao $H$, et al. Analysis of heterosis and quantitative trait loci for kernel shape related traits using triple testcross population in maize. PLoS One. 2015;10:e0124779.

89. Li H, Yang Q, Gao L, et al. Identification of heterosis-associated stable QTLs for ear-weight-related traits in an elite maize hybrid zhengdan 958 by design III. Front Plant Sci. 2017;8:561.

90. Broman KW, Sen Ś. Fit and exploration of multiple-QTL models. In: A guide to QTL mapping with R/qtl. Statistics for biology and health. New York: Springer; 2009. p. 241-82.

\section{Publisher's Note}

Springer Nature remains neutral with regard to jurisdictional claims in published maps and institutional affiliations.
Ready to submit your research? Choose BMC and benefit from:

- fast, convenient online submission

- thorough peer review by experienced researchers in your field

- rapid publication on acceptance

- support for research data, including large and complex data types

- gold Open Access which fosters wider collaboration and increased citations

- maximum visibility for your research: over $100 \mathrm{M}$ website views per year

At BMC, research is always in progress.

Learn more biomedcentral.com/submissions 